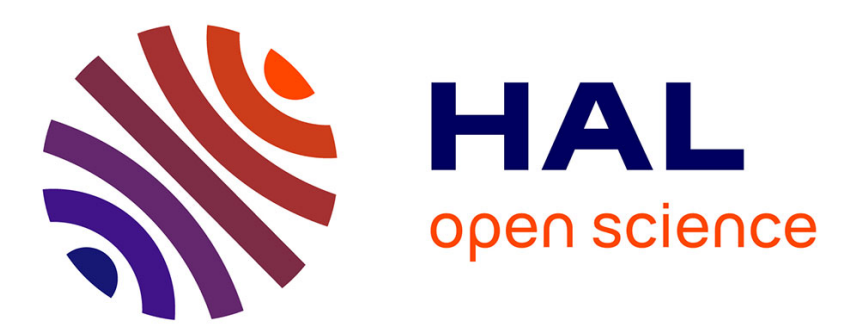

\title{
Problèmes liés au développement des commutateurs à semiconducteur amorphe
}

P. Kumurdjian, J.F. Pere, J.M. Mackowski

\section{To cite this version:}

P. Kumurdjian, J.F. Pere, J.M. Mackowski. Problèmes liés au développement des commutateurs à semiconducteur amorphe. Revue de Physique Appliquée, 1977, 12 (5), pp.697-705. 10.1051/rphysap:01977001205069700 . jpa-00244229

\section{HAL Id: jpa-00244229 https://hal.science/jpa-00244229}

Submitted on 1 Jan 1977

HAL is a multi-disciplinary open access archive for the deposit and dissemination of scientific research documents, whether they are published or not. The documents may come from teaching and research institutions in France or abroad, or from public or private research centers.
L'archive ouverte pluridisciplinaire HAL, est destinée au dépôt et à la diffusion de documents scientifiques de niveau recherche, publiés ou non, émanant des établissements d'enseignement et de recherche français ou étrangers, des laboratoires publics ou privés. 


\title{
PROBLÈMES LIÉS AU DÉVELOPPEMENT DES COMMUTATEURS A SEMICONDUCTEUR AMORPHE
}

\author{
P. KUMURDJIAN, J. F. PERE \\ Commissariat à l'énergie Atomique, Centre d'Etudes de Bruyères-le-Châtel \\ B. P. 561, 92542 Montrouge Cedex, France
}

\section{J. M. MACKOWSKI}

Institut de Physique Nucléaire Université Claude Bernard Lyon-1 43, Boulevard du 11 Novembre 1918, 69621 Villeurbanne, France

\begin{abstract}
Résumé. - Nous passons en revue l'ensemble des problèmes qui nous sont apparus lors de la réalisation de composants de commutation utilisant une couche mince de semiconducteur amorphe du type chalcogénure. Aspects technologiques et comportement thermique ou électrique, notamment diffusion et électromigration des électrodes, sont illustrés par quelques-uns de nos résultats, puis analysés afin d'aboutir à un ensemble de conclusions permettant d'espérer l'apparition de dispositifs satisfaisants, susceptible d'être à la fois le support d'expériences précises sur les semiconducteurs amorphes et un composant fiable pour l'électronique.
\end{abstract}

\begin{abstract}
We review the main difficulties that we encountered in the manufacturing of switching components based on amorphous thin film chalcogenide semiconductor. We discuss the technological, thermical and electrical aspects of the problems, particularly electrodes diffusion and electromigration; some of our results illustrate the discussion. This brings us to the conclusion that it is possible to obtain satisfactory devices in the near future, both for amorphous semiconductor test vehicles, and for reliable electronic components.
\end{abstract}

1. Introduction. - Un domaine d'application particulièrement intéressant des semiconducteurs amorphes en électronique consiste à exploiter leurs propriétés de commutation et de mémorisation. Il est établi que les meilleurs résultats sont obtenus avec des mélanges chalcogénures tels que As-Te-Ge-Si où As-Te-Ge-S. Nous n'aborderons ici que les dispositifs à effet de commutation électronique pur [1] fonctionnant en régime impulsionnel ou continu, avec des courants importants à l'état conducteur.

Disposer d'un composant, mais aussi d'un véhicule test valable, nous paraît fondamental quand on désire étudier les semi conducteurs amorphes. Or il est troublant de découvrir dans les publications l'extraordinaire variété des dispositifs réalisés à des fins expérimentales quantitatives ; corrélativement, nous constatons une grande confusion quant aux effets observés et aux mécanismes proposés concernant la commutation.

Nous allons essayer de passer en revue les problèmes technologiques à résoudre lors de la réalisation d'un dispositif, mais aussi d'analyser les phénomènes qui apparaissent lors de son utilisation. Nous tâcherons d'en tirer quelques réflexions conditionnant la réussite d'un tel composant.

2. Aspects technologiques. - Pour obtenir des tensions de seuil voisinant la dizaine de volts, le semi- conducteur amorphe doit avoir une épaisseur de l'ordre du micromètre. Cette nécessité rend la réalisation épineuse, le comportement d'une couche mince étant extraordinairement plus fin que celui du même matériau sous forme massique.

La fabrication d'un dispositif passera donc par un certain nombre de manipulations classiques : le dépôt des couches minces et la gravure de ces couches.

2.1 GÉOMÉTRIE DU DISPOSITIF. - Il s'agit en premier lieu de définir l'aspect, la structure géométrique de ce composant. Grossièrement, se dégagent deux sortes de composants :

$1^{\text {er }}$ cas: le film de semiconducteur est pris en sandwich entre les deux électrodes :

- une électrode inférieure définie par masquage direct lors du dépôt, partiellement chevauchée par une électrode supérieure également définie, séparées par une couche du semiconducteur à étudier : si l'électrode inférieure est très mince par rapport à l'épaisseur du verre pour éviter l'effet de marche, ce dispositif est satisfaisant pour l'étude des effets thermiques exposés au $\S 2$;

- deux billes, ou un substrat plan et une bille, recouverts d'une couche de semiconducteur et mis en contact: unique solution actuellement satisfaisante pour l'étude de la commutation, malgré la sensibilité 

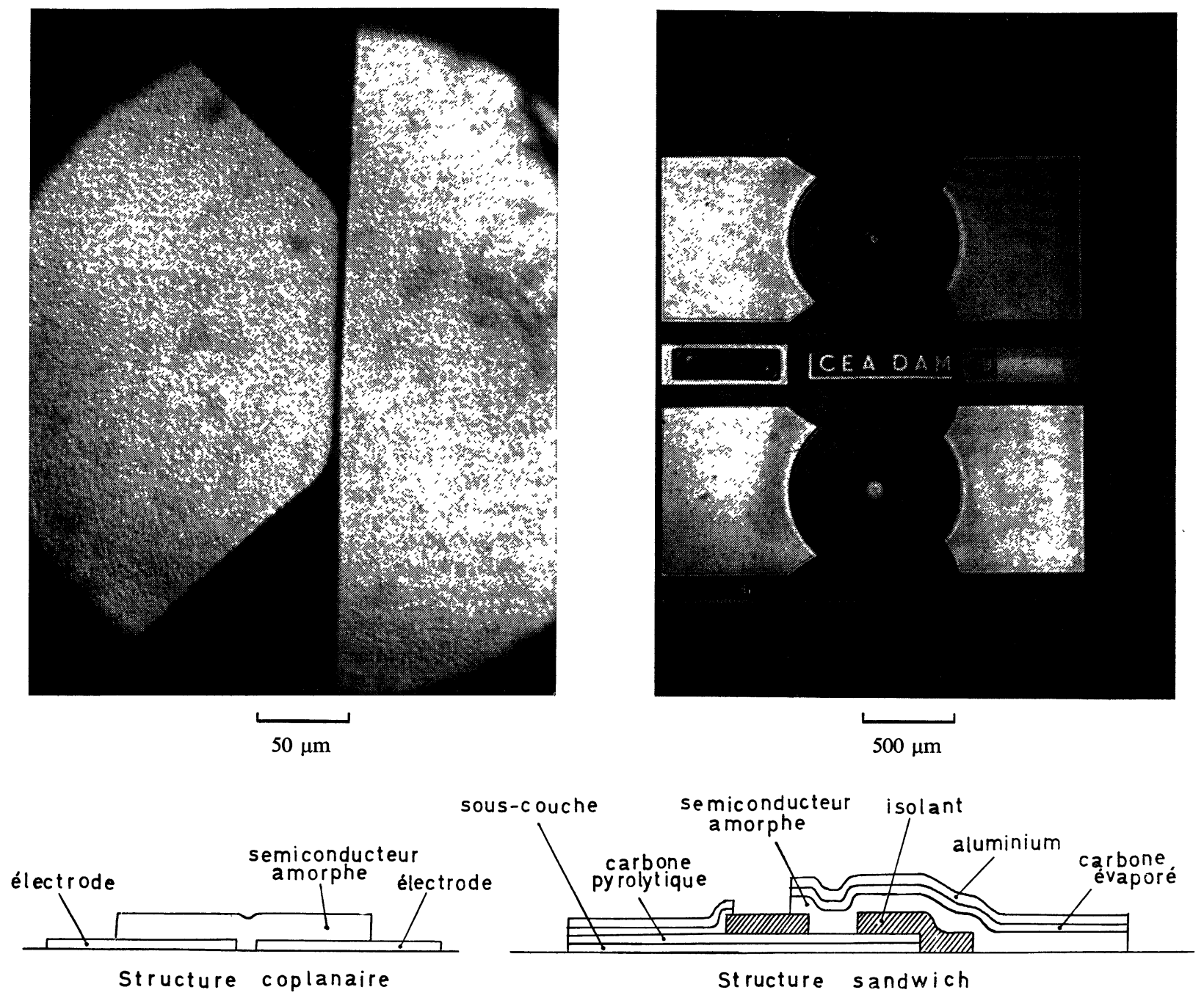

FIG. 1. - Géométries possibles d'un dispositif.

mécanique, l'effet de la pression exercée, et la qualité de l'interface de contact (oxydation, surface active imprécise, etc...) [2] ;

- une véritable structure au sens microélectronique, telle que décrite référence [3], et dont le processus d'obtention est au point (Fig. 1).

$2^{\text {e }}$ cas : le film de semiconducteur est utilisé longitudinalement :

- deux pointes de touche très rapprochées en contact avec la surface du semiconducteur : dispositif hasardeux donnant des résultats entachés d'arte fact ;

- deux électrodes coplanaires séparées par un très fin canal et recouvertes d'une épaisse couche de semiconducteur (Fig. 1).

Nous avons exposé référence [4] les résultats donnés par ces diverses structures. Ceux-ci nous conduisent aux réflexions suivantes :

- la conception de la structure géométrique et le choix des matériaux utilisés doivent fondamentalement être guidés par la connaissance que nous avons actuellement du phénomène commutation, à savoir primordialement son aspect volumique puis filamentaire, ensuite l'aspect évacuation thermique, contraintes mécaniques, et capacité parasite ;

- l'adoption d'une technologie extrêmement propre exempte de certaines contaminations catastrophiques [5].

Les dispositifs coplanaires sont immédiatement éliminés : dissymétrie thermique, partie active en contact avec l'air, section d'électrodes en vis-à-vis trop faible, irrégularité des bords d'électrode.

Parmi les dispositifs sandwich, pour les raisons évidentes de qualité, la troisième structure citée sera retenue, encore que celle-ci doit être optimisée [6].

\subsection{Processus DE RÉAlisation. - 2.2.1 Le dépôt} des couches minces. En premier lieu, il s'agit de savoir réaliser une couche mince de semiconducteur amorphe qui soit une réplique exacte à tout point de vue du même semiconducteur sous forme massique. La pulvérisation cathodique est la solution la moins mauvaise [7] dans le cas des composés chalcogénures. Nous lui reprochons de donner des couches présentant d'une part des défauts lacunaires, d'autre part des piégeages gazeux provenant de la décharge : la porosité entrâ̂- 
nera une évolution de la couche et surtout, nous le verrons, facilitera la diffusion ; les gaz occlus, notamment l'oxygène résiduel, pourront conduire à une dégradation non négligeable; c'est ainsi que des pulvérisations volontairement conduites avec une pression partielle d'oxygène croissante montrent que la résistivité du verre varie et son énergie d'activation chute. Une amélioration actuellement mise en place consiste à utiliser la pulvérisation par faisceau d'ions.

Le dépôt de l'électrode supérieure est l'opération la plus difficile à réussir car il se fait sur le semiconducteur amorphe. Il est prouvé aujourd'hui $[8,9]$ que :

- l'évaporation thermique cristallise une certaine profondeur du semiconducteur;

- la pulvérisation implante littéralement du métal dans une profondeur non négligeable du semiconducteur.

Les conséquences prévisibles sont soit une cristallisation se propageant rapidement dans tout le semiconducteur amorphe à partir de l'amorce créée par l'évaporation, soit une diffusion et une cristallisation initiées par l'implantation du métal pulvérisé. Il faut cependant admettre que l'interface sera toujours perturbé par une interpénétration où une liaison chimique entre les deux matériaux, ne serait-ce que pour assurer une adhérence minimum.

De plus, la nature de l'électrode détermine la technique utilisée. En particulier, le carbone qui est le meilleur choix quant à la qualité du dispositif, conduit à un difficile compromis avec la nécessité d'être conducteur c'est-à-dire d'avoir une structure approximativement graphitée qui ne peut être obtenue qu'à chaud. Pour le molybdène, les difficultés sont de l'avoir suffisamment épais sans décollement - résultat que l'on n'obtient qu'à chaud [10] - et exempt d'oxygène : nous avons relevé par rétrodiffusion $\alpha$-Li et analyse à la sonde ionique jusqu'à $10 \%$ d'oxygène piégé dans la couche. A ce propos, le first-fire pourrait être raisonnablement attribué au claquage de l'oxyde déposé en même temps que l'électrode.

2.2.2 La photogravure. La gravure des couches minces peut être conduite selon plusieurs techniques. Pour des motifs de pollution et de compatibilité [7], la gravure chimique n'a pas été retenue pour les commutateurs. La gravure par érosion mécanique obtenue par un faisceau d'ions [11] paraît mieux adaptée. Elle présente certains dangers : risques de nucléation par impact, décomposition superficielle par pulvérisation préférentielle et échauffement, pollution des surfaces par le support.

2.3 Remarques. - La localisation de la partie active du dispositif est obtenue par une fenêtre dans un isolant (Fig. 1). Le semiconducteur est par conséquent longitudinalement en contact avec cet isolant. Nous avons constaté que si cet isolant est constitué par un oxyde obtenu par pulvérisation réactive, le dispositif obtenu a une durée de vie très faible due à l'oxydation de l'amorphe. Il convient donc, soit d'éviter l'utilisation d'un oxyde - et à cet égard un chalcogénure isolant nous paraît mieux adapté - soit d'obtenir un oxyde sans oxygène libre (dépôt par décomposition thermique).

Nous avons observé qu'un dispositif recouvert d'une couche isolante de protection voit sa cinétique de dégradation accélérée. En conséquence, lors de la conception de la structure géométrique d'un dispositif, il faut au départ prévoir l'isolant permettant de descendre l'électrode supérieure sur le substrat afin de terminer par le dépôt de la partie active constituée par le semiconducteur et l'électrode supérieure (Fig. 1).

3. Phénomènes liés au fonctionnement. - La technologie étant relativement figée, nous disposons de composants permettant de faire des mesures électriques réalistes. Or à ce stade, nous constatons que la durée de vie des composants est courte. Nous obtenons un rendement actuel légèrement supérieur à $1 \%$, un composant étant jugé bon s'il fonctionne parfaitement et réversiblement en continu, avec un courant à l'état conducteur de plusieurs dizaines de milliampères.

Nous allons examiner plus en détail les effets prenant naissance lors de la mise en service du dispositif : la diffusion et l'électromigration des électrodes dans le semiconducteur.

3.1 LA DIFFUSION THERMIQUE. - La diffusion thermique à l'interface métal-semiconducteur a été étudiée à une température inférieure à $T_{\mathrm{g}}$, en choisissant l'interface or-semiconducteur amorphe $[8,12]$ particulièrement propice aux évaluations.

Pour suivre la dégradation du matériau avec la diffusion, nous avons choisi le paramètre énergie d'activation $\Delta E$ de la résistivité $\rho$ afin de s'affranchir des imprécisions de la structure sandwich utilisée

La figure $2 a$ donne le pourcentage $\tau$ de variation $\Delta E$ en fonction du temps pour plusieurs températures de recuit.

La figure $2 b$ donne le profil de concentration d'or au milieu du semiconducteur, en fonction du temps, relevé par rétrodiffusion pour ces mêmes recuits.

La figure $2 c$ donne la corrélation entre la dégradation et la concentration.

\section{L'examen de ces courbes montre :}

- l'existence d'une concentration d'or non-nulle $(0,1$ à $0,3 \%)$ dans tout le semiconducteur, traduisant une diffusion presque instantanée mais de faible amplitude ;

- l'apparition subite d'une forte concentration d'or correspondant à la rapide dégradation du dispositif.

On constate le caractère très particulier de cette diffusion dans les mélanges vitreux chalcogénures puisqu'elle ne suit pas les lois de Fick alors que celles-ci 

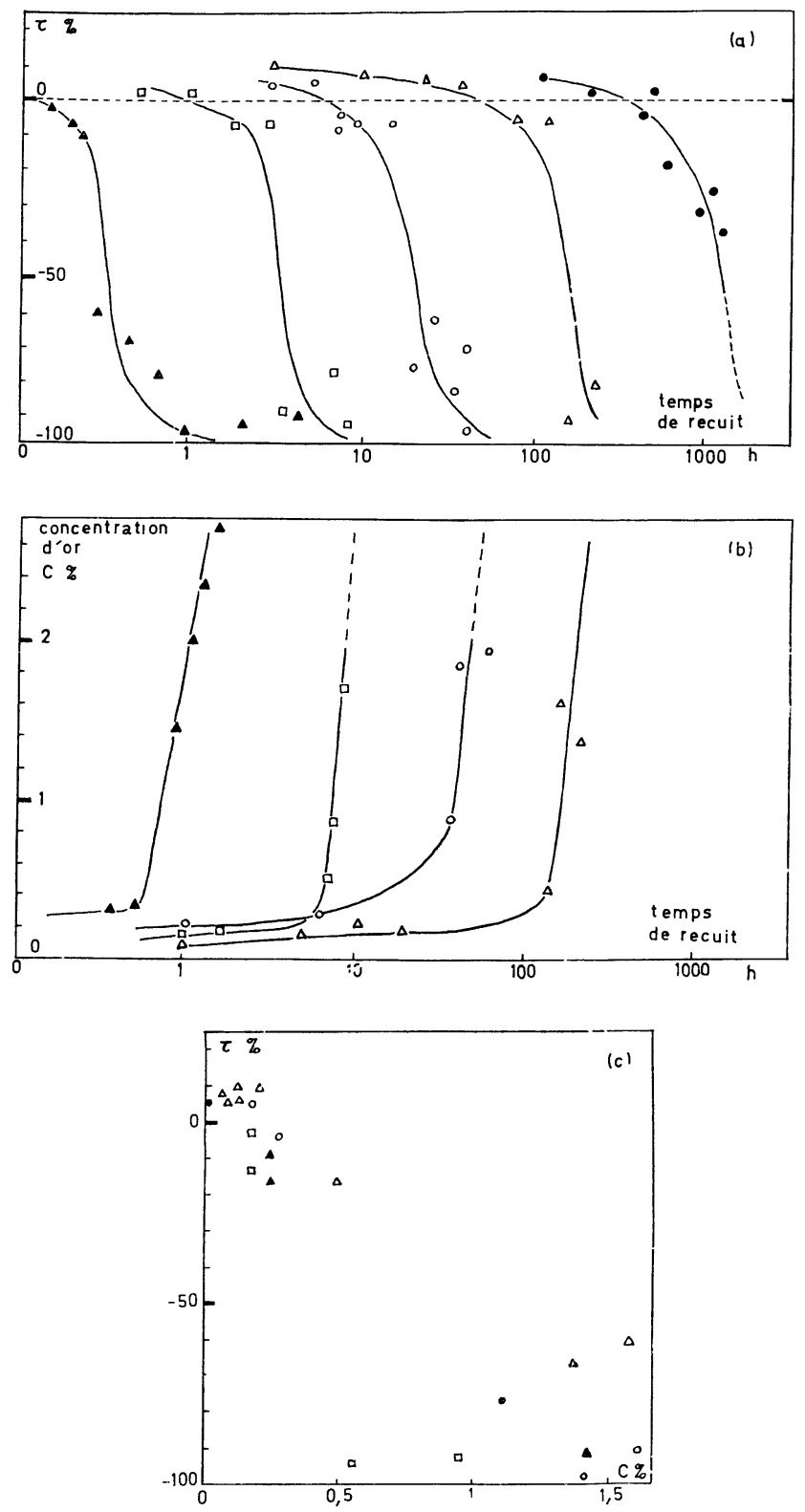

Fig. 2. - Dégradation $\tau$ de l'énergie d'activation par diffusion thermique pour une structure Or/As ${ }_{38} \mathrm{Te}_{43} \mathrm{Ge}_{14} \mathrm{~S}_{5} / \mathrm{Or}$.

Température de recuit : $\Delta 190^{\circ} \square 170^{\circ} \bigcirc 150^{\circ} \triangle 120^{\circ}$ - $100^{\circ} \mathrm{C}$.

sont vérifiées pour le système or-arsenic amorphe évaporé : figure 3.

Plusieurs hypothèses peuvent être avancées pour expliquer ce comportement.

La diffusion instantanée peut être due à l'existence de canaux (probablement des zones lacunaires de moindre compacité dans le semiconducteur et inhérentes au dépôt par pulvérisation cathodique, ou bien des failles qui caractérisent souvent les verres) autorisant un coefficient de diffusion très supérieur à celui du matériau massique.

Une autre hypothèse met en jeu la structure même du semi conducteur. Nous avons montré [13] qu'en réalité les amorphes du type chalcogénure sont constitués d'un édifice moléculaire de base défini formant des
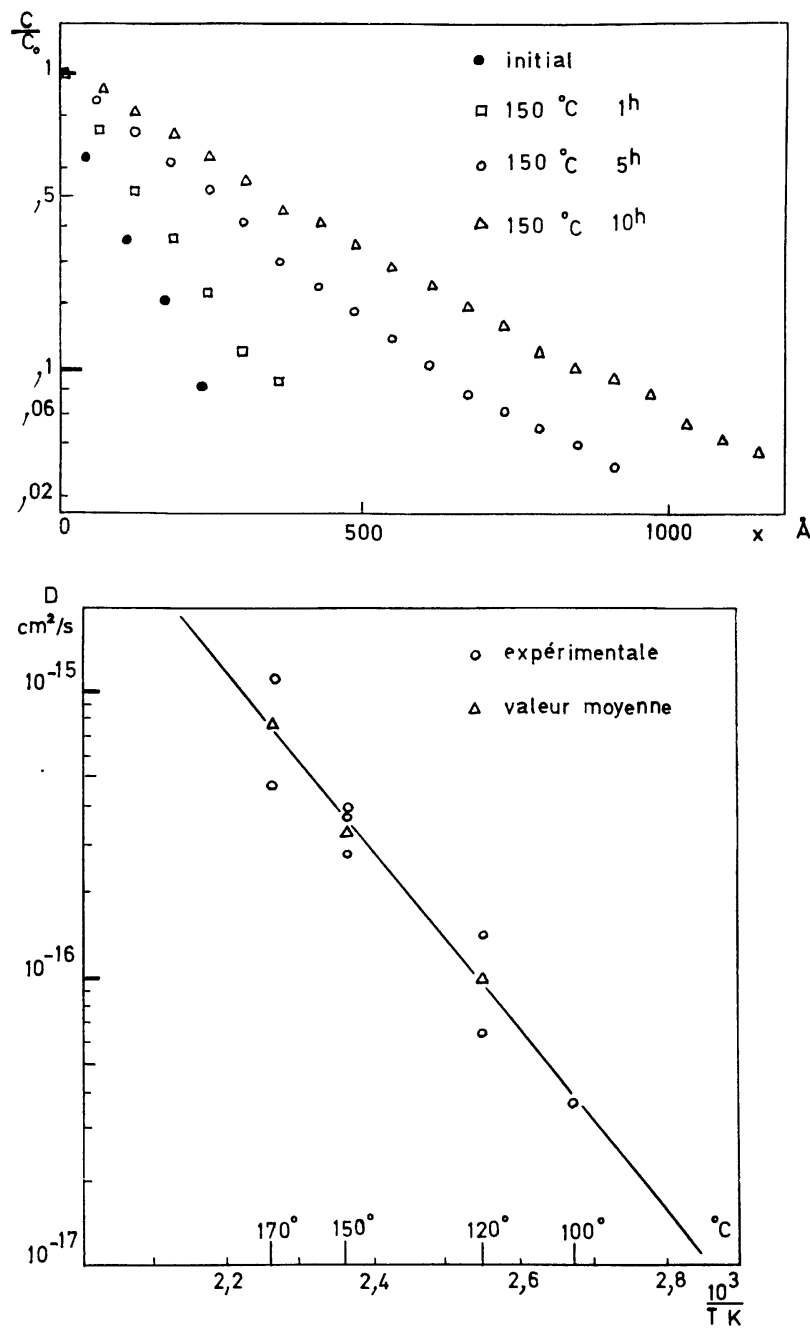

Fig. 3. - Profil de diffusion $\frac{C}{C_{0}}=\operatorname{erfc} \frac{x}{2 \sqrt{D t}}$ pour une structure or/arsenic.

entités liées entre elles par des zones perturbées constituées par les autres éléments en excès. Ces dernières régions pourraient être propices à une migration de masse.

On notera que Biktimirova et al. [14] avancent que ces deux types de diffusion sont dus à la position de l'or dans le verre : liaison covalente avec les composants du verre ou position interstitielle.

$\mathrm{La}$ dégradation électrique du dispositif peut se comprendre de plusieurs manières :

- soit par la cristallisation du semiconducteur, l'or jouant le rôle de centre nucléant dans le verre $[12,15]$;

- soit par l'apparition d'une conductivité métallique due à ce que les états localisés créés dans le gap électrique par l'or deviennent une bande quand la concentration d'or augmente brusquement.

Il est important de prendre en considération la dégradation des caractéristiques thermiques du semiconducteur avec le recuit. Il est surprenant de constater que les températures caractéristiques $T_{\mathrm{g}} T_{\mathrm{c}}$ et $T_{\mathrm{f}}$ existent 
toujours avec la même amplitude et la même valeur, y compris quant le dispositif est totalement dégradé électriquement (cas du verre AsTe avec l'or). Ceci sousentend que si l'on a cristallisation, celle-ci reste suffisamment faible après dégradation pour ne pas être décelable en A.T.D.

A partir des résultats concernant la dégradation électrique par diffusion, il est intéressant du point de vue dispositif, d'extrapoler la durée de vie avec la température (Fig. 4). On voit de façon explicite qu'un dispositif ayant atteint $T_{\mathrm{g}}$ même pendant un temps très court, ne peut fonctionner correctement. On peut juger ici de l'importance que prend l'obtention d'une commutation électronique et non thermique sur la fiabilité des dispositifs.

$\begin{array}{cc}\text { Température } \theta^{\circ} \mathrm{C} & \text { Durée de vie } \\ \overline{20} & - \\ 30 & 1825 \text { ans } \\ 50 & 411 \text { ans } \\ 60 & 26 \text { ans } \\ 100 & 5 \text { ans } \\ 150 & 5 \text { semaines } \\ 190 & 12 \mathrm{~h} \\ T_{\text {gmax }} & \sim 1 \mathrm{~h} \\ \end{array}$

Fig. 4. - Durée de vie en fonction de la température pour une structure $\mathrm{Or} / \mathrm{As}_{38} \mathrm{Te}_{43} \mathrm{Ge}_{14} \mathrm{~S}_{5} / \mathrm{Or}$.

Des recuits à $T_{\mathrm{g}}$ montrent la formation de microcristaux riches en Te. De plus, des verres mal choisis présentent des pertes, notamment en arsenic.

Si l'on s'éloigne du cas des électrodes en or, on constate toujours les mêmes phénomènes de diffusion, mais avec une cinétique différente. La figure 5 montre l'effet de la nature des électrodes sur la durée de vie pour une dégradation de $20 \%$. Ainsi pour le molybdène, la diffusion est dix fois plus lente. Des observations en cours, combinant des résultats d'analyse à la sonde ionique et à la sonde de Castaing, semblent montrer que le molybdène diffuse rapidement dans les parties du semiconducteur amorphe contenant de l'oxygène, soit par contact de l'amorphe avec le Mo (qui contient toujours de $\mathrm{l}^{\prime} \mathrm{O}_{2}$ ), soit par l'air ambiant.

Dans le cas du carbone, nos premiers résultats montrent qu'aucune dégradation décelable n'apparaît.

3.2 L'éleCtromigration. - L'application d'un champ électrique sur un dispositif est une contrainte favorisant l'électromigration [16].

Avant d'aborder ce sujet, nous devons signaler que l'application d'un champ électrique continu de l'ordre de $1 \times 10^{-4} \mathrm{~V} / \mathrm{cm}$ lors d'un recuit (parfaitement contrôlé en bain d'heptane) provoque un échauffement supplémentaire non négligeable du semiconducteur amorphe. Le tableau figure 6 compare la durée de vie donnée figure 4 sans champ et celle observée ici avec champ à même température de recuit. On arrive à évaluer à environ $18^{\circ} \mathrm{C}$ l'échauffement supplémentaire dû au champ électrique.

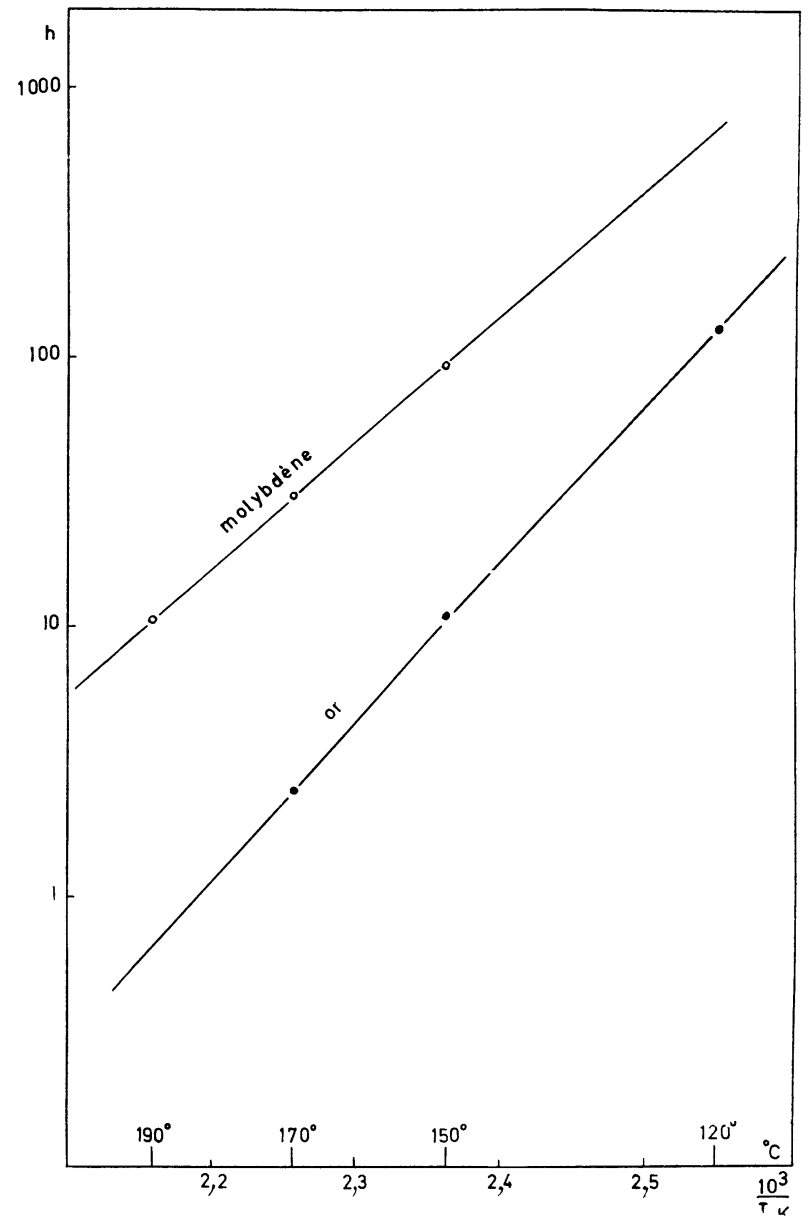

Fig. 5. - Comparaison des durées de vie pour les structures Or/As ${ }_{38} \mathrm{Te}_{43} \mathrm{Ge}_{14} \mathrm{~S}_{5} / \mathrm{Or}$ et Mo/As ${ }_{38} \mathrm{Te}_{43} \mathrm{Ge}_{14} \mathrm{~S}_{5} / \mathrm{Mo}$.

$\begin{array}{ccccc}\begin{array}{c}\text { Tem- } \\ \text { péra- } \\ \text { ture }\end{array} & \begin{array}{c}\text { Durée de vie } \\ \text { calculée figure } 4 \\ \text { sans champs } \\ \text { électrique }\end{array} & \begin{array}{c}\text { Durée de vie } \\ \text { observée } \\ \text { avec champs } \\ \text { électrique }\end{array} & \begin{array}{c}\text { Variation } \\ \text { relative }\end{array} & \Delta \theta \\ - & - & - & - & - \\ 70^{\circ} \mathrm{C} & 2,14 \times 10^{4} \mathrm{~h} & 750 \mathrm{~h} & 96 \% & 18,9^{\circ} \mathrm{C} \\ 85^{\circ} \mathrm{C} & 3,97 \times 10^{3} \mathrm{~h} & 320 \mathrm{~h} & 92 \% & 17,9^{\circ} \mathrm{C}\end{array}$

Fig. 6. - Elévation de température $\Delta \theta$ du verre $\mathrm{As}_{38} \mathrm{Te}_{43} \mathrm{Ge}_{14} \mathrm{~S}_{5}$ due à l'application d'un champ électrique de $1 \times 10^{4} \mathrm{~V} \cdot \mathrm{cm}^{-1}$.

L'observation visuelle de l'aspect des électrodes lors du recuit montre à l'évidence un transport de l'or de l'électrode positive vers l'électrode négative. Ce transport est réversible si l'on change la polarité avant destruction par coupure : figure 7. Enfin un dispositif polarisé en alternatif ne présente pas ces migrations. Les spectres de rétrodiffusion confirment parfaitement ces observations.

On remarquera que cette migration impliquerait que l'or a une charge positive, donc une position interstitielle [17], et n'est pas conforme à la direction de l'électromigration trouvée dans les publications [18]. 


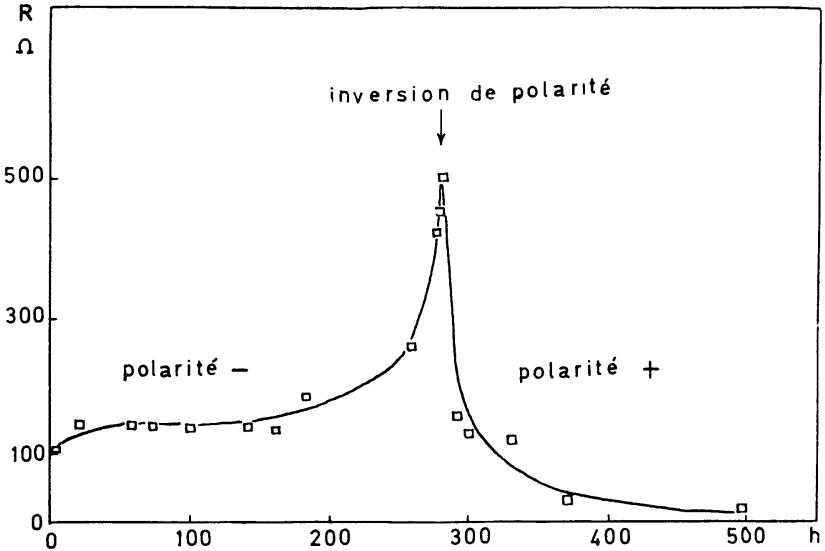

Fig. 7. - Résistance d'un dispositif en fonction du temps et de la polarité de l'électrode supérieure, pour un champ électrique de $1 \times 10^{4} \mathrm{~V} \cdot \mathrm{cm}^{-1}$.

3.3 EfFets SUR LA COMmUtation. - Les dispositifs utilisés comportent un substrat isolant sur lequel sont successivement déposés l'électrode inférieure et le semiconducteur amorphe, l'électrode supérieure étant constituée par l'application d'une demi-sphère de carbone poli de $4 \mathrm{~mm}$ de diamètre. La figure 8 montre l'évolution de la résistance du dispositif en fonction du nombre d'impulsions dans le cas de l'or comme électrode inférieure.

La figure 9 montre l'évolution de la tension de commutation avec le nombre d'impulsions pour des

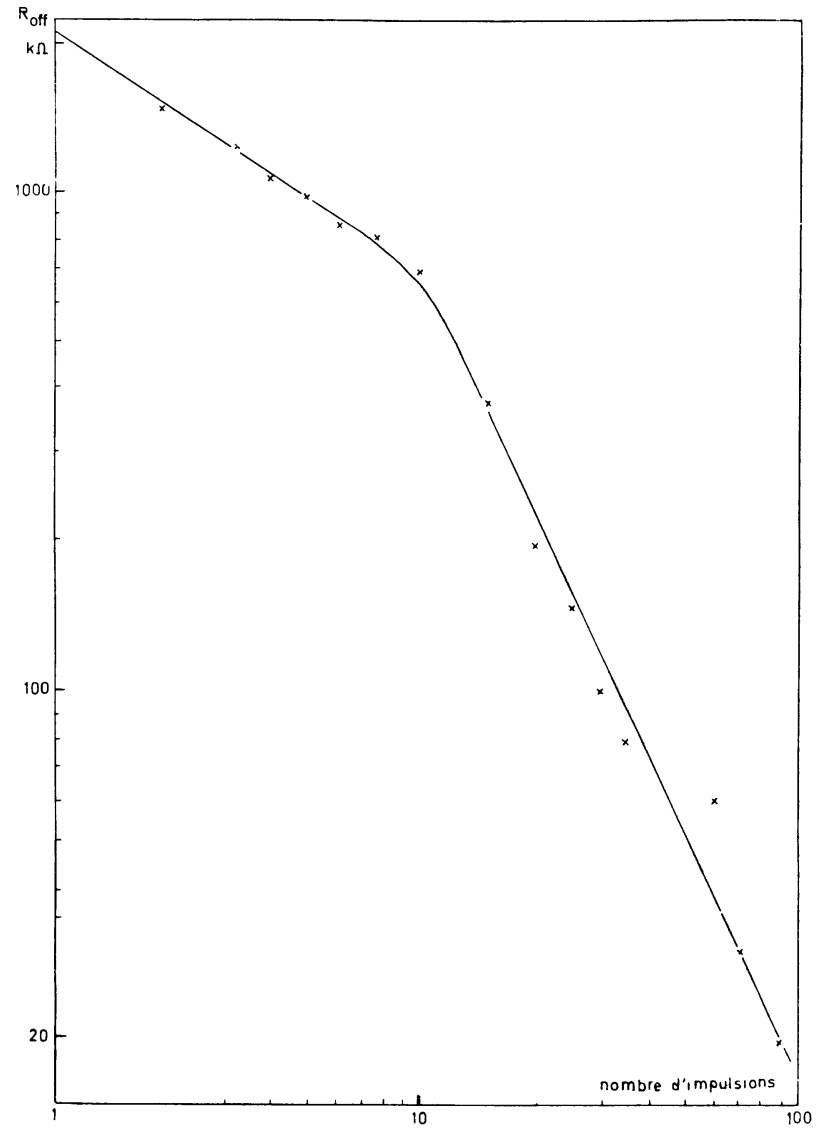

FIG. 8. - Résistance $R$ d'un dispositif $\mathrm{Or} / \mathrm{As}_{25} \mathrm{Te}_{61} \mathrm{Ge}_{13} \mathrm{~S}_{1} /$ carbone après chaque impulsion de commutation.

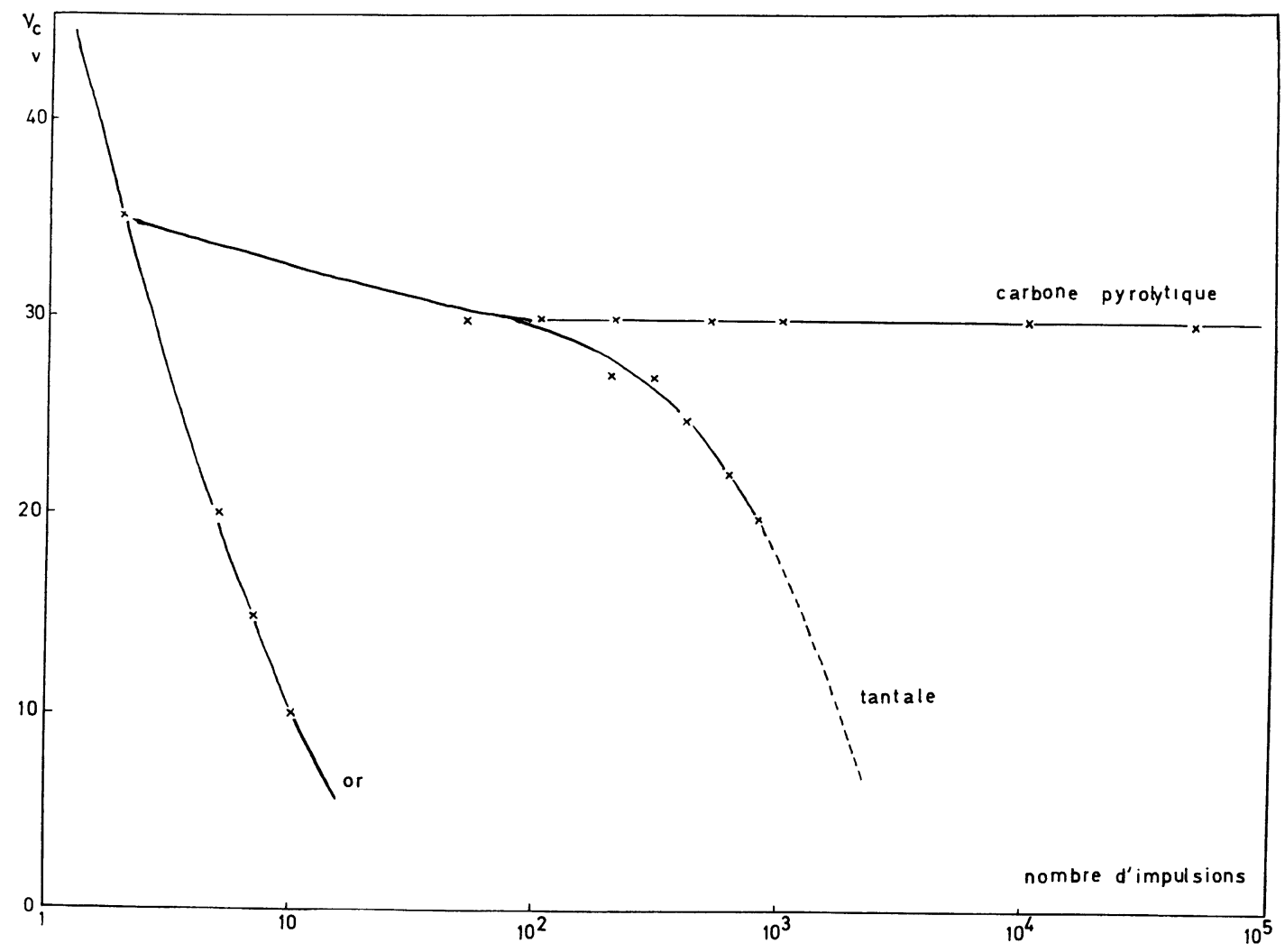

FIG. 9. - Tension $V_{\mathrm{c}}$ de commutation pour le verre $\mathrm{As}_{25} \mathrm{Te}_{61} \mathrm{Ge}_{13} \mathrm{~S}_{1}$ à chaque impulsion en fonction de la nature des électrodes. 
électrodes inférieures de nature différente. On ajoutera que ces durées de vie sont d'autant plus courtes que le courant commuté est grand.

4. Discussion. - 4.1 LA NOTION D'IMPURETÉ. Tous ces résultats ont montré une circulation du métal électrode dans le semiconducteur amorphe lorsque le dispositif est soumis à un recuit ou à un champ électrique : la présence de métal par diffusion solide-solide altère les propriétés électriques alors qu'il était admis que les semiconducteurs amorphes étaient insensibles aux impuretés.

Nous avons préparé des lingots de verre $\mathrm{As}_{50} \mathrm{Te}_{50} \mathrm{Au}_{x}$. Nous avons remarqué que pour $x=0,050,5$ et $5 \%$, les caractéristiques du verre ne sont pas affectées de façon significative. Des spectres E.S.R. sur ces composés sont parfaitement plats. Tout ceci indique que l'or s'est parfaitement intégré dans le semiconducteur et que nous avons affaire à un matériau covalent. Des lingots de verre $\mathrm{As}_{34} \mathrm{Ge}_{16} \mathrm{Te}_{28} \mathrm{~S}_{22}$ ont été préparés avec de l'aluminium à $0,10,5$ et $1 \%$. Ici, de légères variations sont constatées, surtout sur la résistivité : figure 10 .

$\begin{array}{cccc} & T_{\mathrm{g}}{ }^{\circ} \mathrm{C} & \rho_{20^{\circ}} \Omega \mathrm{cm} & \Delta E \mathrm{eV} \\ \mathrm{Te}_{28} \mathrm{As}_{34} \mathrm{Ge}_{16} \mathrm{~S}_{22} & 180-235 & 3.10^{7} & - \\ +0,1 \% \mathrm{Al} & 167-246 & 4.10^{7} & 1,17 \\ +0,5 \% \mathrm{Al} & 182-233 & 8.10^{6} & 1,12 \\ +1 \% \mathrm{Al} & 185-247 & 4.10^{6} & 1,13\end{array}$

Fig. 10. - Influence de l'introduction d'aluminium dans $\mathrm{As}_{34} \mathrm{Te}_{28} \mathrm{Ge}_{16} \mathrm{~S}_{22}$ lors de sa synthèse au four.

Il convient donc de revenir sur la notion d'impureté dans les semiconducteurs amorphes, car selon la manière dont celle-ci est introduite, soit lors de la synthèse, soit lors du dépôt en couche mince, soit lors d'une diffusion ou d'une électromigration, les caractéristiques et le comportement du verre seront considé- rablement différents (nature des liaisons de l'impureté avec les composants du verre, ou de sa précipitation en interstitiel).

4.2 ConséQuences PRATiQues. - La réussite de la fabrication d'un dispositif nous paraît être actuellement basée sur les réflexions suivantes :

1) Le semiconducteur amorphe choisi doit être stable : la matrice vitreuse doit être homogène, bien définie et liée à l'optimum [19].

2) La couche semiconductrice doit être la plus parfaite possible.

3) Les électrodes doivent être convenablement choisies et déposées :

a) Le choix du matériau d'abord, qui doit être le moins diffusant possible et ne pas créer de sites de nucléation dans le semiconducteur amorphe; à cet égard, plusieurs attitudes sont possibles :

- soit on ne veut plus de diffusion et le carbone paraît alors tout indiqué; nous pensons que cette solution n'est pas réaliste, d'autant que le carbone n'est pas suffisamment conducteur pour être employé seul comme électrode supérieure ;

- soit on empêche la diffusion et alors on dispose entre le semiconducteur amorphe et l'électrode une mince couche d'un matériau qui formera barrière; nous avons essayé avec une électrode d'or et une barrière de $200 \AA$ de carbone, et nous avons constaté une dégradation moindre, ainsi qu'elle apparaît figure 11 ; la dégradation de $20 \%$ est due à un léger passage d'or au départ (confirmé par rétrodiffusion);

- soit on accepte la diffusion et on interpose une couche tampon entre l'électrode et le semiconducteur actif. Le rôle de cette couche est d'absorber le plus possible les atomes diffusants et d'éviter ainsi une perturbation de la partie active, et peut-être aussi de fournir de l'autre côté les éléments nécessaires à l'obturation des canaux du semiconducteur. Nous avons

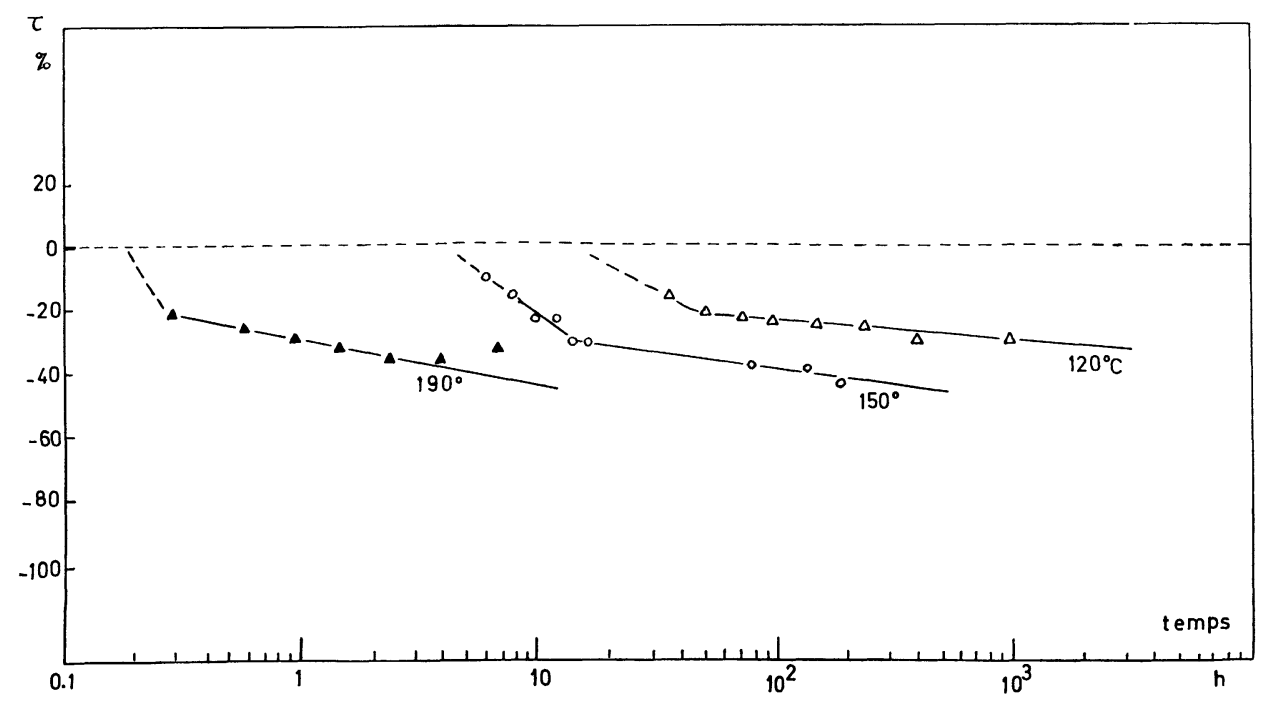

Fig. 11. - Variation $\tau$ en $\%$ de l'énergie d'activation du verre $\mathrm{As}_{38} \mathrm{Te}_{43} \mathrm{Ge}_{14} \mathrm{~S}_{5}$ en fonction du recuit. 
avec succès employé $200 \AA$ de verre $\mathrm{As}_{25} \mathrm{Te}_{61} \mathrm{Ge}_{13} \mathrm{~S}_{1}$ sur une couche active de $1 \mu \mathrm{m}$ de $\mathrm{As}_{34,5} \mathrm{Te}_{27,9} \mathrm{Ge}_{15,6} \mathrm{~S}_{22}$ sans modification des performances. Cette couche tampon a l'avantage également de prendre pour elle les défauts introduits lors du dépôt de l'électrode supérieure. Enfin, il est à remarquer l'effet de la polarité des électrodes [20] pour lequel l'électrode supérieure devrait être la cathode puisqu'étant la plus éprouvante.

b) la technologie de dépôt de l'électrode supérieure doit être la moins perturbante possible.

4) Les couches doivent être exemptes de toute contamination, tout particulièrement de l'oxygène. La figure 12 donne un exemple de l'importance de cette contamination sur le verre $\mathrm{As}_{27} \mathrm{Te}_{33} \mathrm{Ge}_{20} \mathrm{~S}_{20}$.

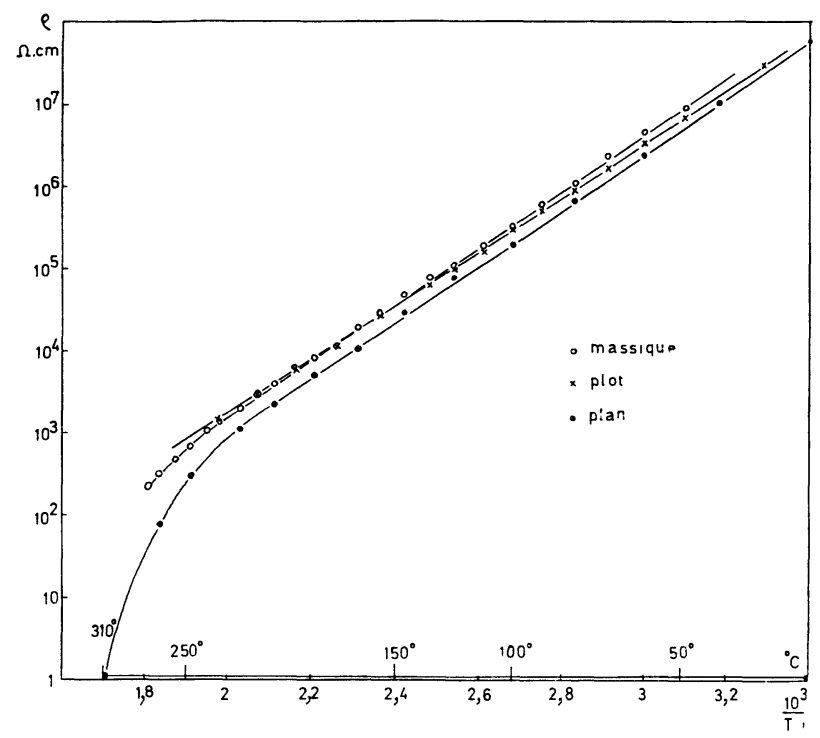

FIG. 12. - Diagramme d'Arrhénius de la résistivité du verre $\mathrm{As}_{27} \mathrm{Te}_{33} \mathrm{Ge}_{20} \mathrm{~S}_{20}$ en fonction de sa présentation.

A l'état massique, ce verre présente une courbe $(\rho T)$ parfaitement réversible de 20 à $310^{\circ} \mathrm{C}$. Deux heures à $280^{\circ} \mathrm{C}$ ne changent aucune caractéristique (rappelons que pour ce verre, la température de transition vitreuse $T_{\mathrm{g}}$ est de $\left.221^{\circ} \mathrm{C}\right)$. Le verre reste amorphe jusqu'à $370^{\circ} \mathrm{C}$ et sa courbe d'ATD est indéfiniment réversible jusqu'à $450^{\circ} \mathrm{C}$. De même une couche mince de ce verre mise en sandwich entre deux électrodes est réversible de 20 à $35^{\circ} \mathrm{C}$ et se conserve même après un recuit de $320^{\circ} \mathrm{C}$. Par contre, cette même couche mais utilisée entre deux électrodes coplanaires n'est plus réversible à partir de $230^{\circ} \mathrm{C}$ et se fixe à $1 \Omega$.cm à $300^{\circ} \mathrm{C}$. La couche étant exposée à l'air s'est oxydée [17]. Cette contamination par l'oxygène est à éviter et le verre semiconducteur doit être protégé de l'air par son électrode supérieure, ne pas être en contact avec des matériaux contenant de l'oxygène libre, et ne pas absorber l'oxygène piégé par le métal d'électrode (cas du molybdène).

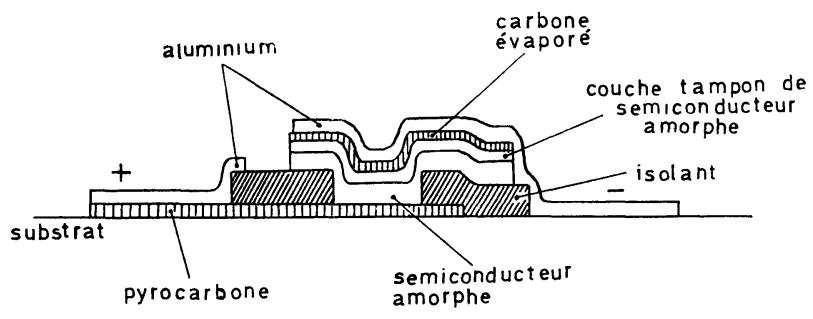

FIG. 13. - Structure proposée d'un commutateur.

5. Conclusion. - Dix ans de travaux sur les semiconducteurs amorphes n'ont pas encore débouchés sur des produits industriels utilisant l'effet de commutation. Ce constat s'explique par le manque d'études technologiques et la méconnaissance de certains phénomènes physiques. Nos résultats montrent que la diffusion et l'électromigration sont un comportement général des dispositifs dont seule la cinétique est modifiée par la nature des électrodes. Ils montrent ensuite l'importance de la maîtrise d'une technologie propre, et aussi les dangers que certaines méthodes font courir. En l'état actuel de nos études, la structure décrite figure 13 peut être considérée comme étant la meilleure à proposer pour la réalisation d'un composant de commutation.

\section{Bibliographie}

[1] Par exemple : Ovshinsky, S. R., Phys. Rev. Lett. 21 (1968) 1450.

Lee, S. H., Henisch, H. K., Solid State Electron. 16 (1973) 155.

Buckley, W. D., Holmberg, S. H., Solid State Electron. 18 (1975) 127.

[2] Neale, R. G., J. Non-Cryst. Solids 2 (1970) 558.

[3] Neale, R. G., Nelson, D. L., Moore, G. E., Electronics 43 (1970) 56

Dargan, C. L., Burton, P., Redstall, R. M., Inter. J. Electron. 38 (1975) 711.

[4] Kumurdian, P., Mackowski, J. M., Samuelli, J. J., 5th International Conference on Amorphous and Liquid Semiconductors (Garmisch Partenkirchen) Septembre 1973.
[5] Ovshinsky, S. R., Fritzsche, H., IEEE Trans. Electron. Devices 20 (1973) 91.

Le Comber, P. G., Loveland, R. J., Spear, W. E., VaUGHAN, R. A., 5th International Conference on Amorphous and Liquid Semiconductors (Garmisch Partenkirchen) Septembre 1973.

[6] Thornburg, D. D., J. Non-Cryst. Solids 17 (1975) 9.

Petersen, K. E., Adler, D., J. Appl. Phys. 47 (1976) 256.

[7] Kumurdian, P., Mackowski, J. M., Samueli, J. J., $1^{\text {re }}$ Conférence C. I. P. 73, Montpellier, Octobre 1973.

[8] Mackowski, J. M., Marsaud, S., Thomas, J. P., KumurdJIAN, P., Conférence C. I. P. 76, Nice, 18-21 Mai 1976.

[9] WeY, H. Y., Phys. Rev. B 13 (1976) 3495.

[10] Rodriguez, A., Misra, M., Hesselbom, Hj., Tove, P. A., Solid State Electron. 19 (1976) 17. 
[11] Pere, J. F., 4 Congrès AVISEM, Toulouse, Octobre 1974.

[12] Mackowski, J. M., Marsaud, S., Tousset, J., KumurdJIAN, P., 6th International Conference on Amorphous and Liquid Semiconductors. Leningrad, 18-24 Novembre 1975.

[13] Mackowski, J. M., Samueli, J. J., Kumurdian, P., Bourdarios, G., J. Non-Cryst. Solids 8-10 (1972) 985.

Mackowski, J. M., Kumurdjian, P., Tousset, J., 3th International Conference on Thin Films, Budapest, Août 1975. Etudes non publiées.

[14] Biktimirova, V. Kh., Boltaks, B. I., Borisova, Z. U.,
Dzhafarov, T. D., Obraetson, A. A., Sov. Phys. Semicond. 8 (1975) 1412.

[15] Yurlova, G. A., Arutyunov, V. S., Kudravtseva, T. N., Inorg. Matér. 9 (1973).

[16] Suptitz, P., Telton, J., Lebedev, E. A., Willert, I., Phys. Stat. Sol. (a) 31 (1975) 31.

[17] Levy, A. W., Green, M., Harley, R. T., J. Phys. C, 7 (1974) L 126.

[18] Blech, I. A., Rosenberg, R., J. Appl. Phys. 46 (1975) 579.

[19] Etudes non publiées sur As-Te-Ge-S.

[20] Petrillo, G. A., KaO, K. C., J. Non-Cryst. Solids 16 (1974) 247. 